\title{
3D-QSAR and docking studies of pentacycloundecylamines at the sigma-1 (o1) receptor
}

Werner J. Geldenhuys, Nicholas Novotny, Sarel F. Malan and Cornelis J. Van der Schyf

\begin{abstract}
Pentacycloundecylamine (PCU) derived compounds have been shown to be promising lead structures for the development of novel drug candidates aimed at a variety of neurodegenerative and psychiatric diseases. Here we show for the first time a $3 \mathrm{D}$ quantitative structure-activity relationship (3D-QSAR) for a series of aza-PCU-derived compounds with activity at the sigma-1 ( $\mathrm{r}_{1}$ ) receptor. A comparative molecular field analysis (CoMFA) model was developed with a partial least squares cross validated $\left(\mathrm{q}^{2}\right)$ regression value of 0.6 , and a non-cross validated $\mathrm{r}^{2}$ of 0.9. The CoMFA model was effective at predicting the sigma-1 activities of a test set with an $\mathrm{r}^{2}>0.7$. We also describe here the docking of the PCU-derived compounds into a homology model of the sigma-1 ( $\left.\mathrm{r}_{1}\right)$ receptor, which was developed to gain insight into binding of these cage compounds to the receptor. Based on docking studies we evaluated in a $[3 \mathrm{H}]$ pentazocine binding assay an oxa-PCU, NGP1-O1 $\left(\mathrm{IC}_{50}=1.78 \mathrm{lM}\right)$ and its phenethyl derivative $\left(\mathrm{IC}_{50}=\right.$ 1.54 $\mathrm{lM}$ ). Results from these studies can be used to develop new compounds with specific affinity for the sigma-1( $\left.\mathrm{r}_{1}\right)$
\end{abstract}

Sigma receptors were initially thought to belong to the opiate receptor family, but were later shown to better associate with another unique family of small proteins. ${ }^{1}$ These receptors are now divided into two subtypes denoted as sigma-1 ( $\mathrm{r}_{1}$ ) and sigma-2 $\left(\mathrm{r}_{2}\right) .2,3$ These relatively small receptors act as chaperone molecules where, upon agonist activation, they are able to translocate from the endoplasmic reticulum (ER) to the cell membrane. 4 The two main sigma receptors were found to differ in size, the sigma-1 receptor being a rv29 $\mathrm{kDa}$ single polypeptide5 and the sigma-2 receptor ranging in size between 18 and $21.5 \mathrm{kDa} .6$ Additionally, the sigma-1 receptor has been cloned, whereas the sigma-2 receptor has not been cloned to date.5,6 The sigma receptor signaling pathway still remains to be fully elucidated but current literature suggests that the sigma receptor protein plays a role in cellular stress by modulation of calcium flux in the ER as well as mitochondria.7,4,8,9 Recently, it has been shown in 
sigma-1 receptor knock-out mice that the mice showed signs of accelerated retinal ganglion cell death, suggesting that sigma receptor ligands may have possible utility in preventing the neurodegeneration seen in glaucoma. ${ }^{10}$ These findings corroborate previous work which suggested that $(+)$-pentazocine afforded neuroprotection against retinal neurodegeneration via sigma-1 receptors. ${ }^{11}$

In the late 1990s, it was shown that pentacycloundecylamine-derived compounds (PCUs) exhibit affinity for the sigma-1 and sigma-2 receptors. This class of polycyclic cage compounds also was shown to display selectivity between the two sigma receptors. ${ }^{12}$ Furthermore, pentacycloundecylamine-derived compounds have been shown to have functional activity on the sigma receptor system, such as enhancing the dopamine releasing effect of amphetamine ${ }^{13}$ as well as methamphetamine, ${ }^{14}$ and also were able to modulate addiction behavior with cocaine via sigma receptor modulation. 15 Binding studies have shown that for sigma-1 receptor binding, an ethyllinker is needed between the aza-bridgehead and the aromatic ring, whereas for sigma-2, a benzyl moiety is more favorable. ${ }^{16}$ Additionally, meta-substitution on the aromatic ring favors increased affinity for both of these receptors, with the halogen fluorine appearing to afford optimal activity. ${ }^{16}$

In our efforts to develop neuroprotective compounds in glaucoma, we focused on gaining insight into the structure-activity relationships of the PCU compounds at the sigma-1 receptor. To date, no $3 \mathrm{D}$ quantitative structure-activity relationship (3DQSAR) studies have been published for pentacycloundecylamine-derived compounds. The current study aims to elucidate the steric and electrostatic features for binding to sigma-1 receptors, which may aid medicinal chemists to design and optimize pentacycloundecylamine-derived compounds as sigma ligands. $3 \mathrm{D}-\mathrm{QSAR}$ modeling was done using the CoMFA modules of SYB-YL 8.1 (Tripos, St. Louis, MO), running on a Dell XPS720 3.66 GHz PC dual booted to run Red Hat Linux Enterprise 5 and Microsoft Windows XP. Compound alignment for CoMFA was performed using the software suite from Openeye (Santa Fe, NM, USA). Compounds were drawn in SYBYL, and exported as SDF files. The SDF files were then used by OMEGA 2.3.2 (OpenEye Scientific Software, Santa Fe, NM, http://www.eyesopen.com) which generates multiple conformers of each compound. ROCS 2.4.1 (OpenEye Scientific Software, Santa Fe, NM, http://www.eyesopen.com) (commandline mode) software from Openeye using the RANKBY COMBO flags which optimize both shape overlay and chemical (color) overlay. 27,28 Compound 5 was used as query. The resulting overlay of compounds shown in Table 1 was then used for the CoMFA analysis. The aligned compounds (Fig. 1) from ROCS were used for the study. Gasteiger-Hückel charges were added before the CoMFA calculation. The compounds used to develop the CoMFA model were divided into a training set and a test set using the QSAR Wizard program for MOE, available for download at the Chemical Computing group SVL exchange (http://svl.chemcomp.com/). The complete set was 
randomly divided irrespective of activity or chemical composition, so that an $80 \%$ separation could be achieved. 23

Default values provided in the Tripos CoMFA module were used with a $2.0 \AA$ grid spacing using an sp3 carbon atom with a +1 point charge as a probe to explore the steric and electrostatic interactions at the lattice points in the grid. The default cut-off value was set at $30 \mathrm{kcal} / \mathrm{mol}$. Statistical analysis was performed using the partial least squares method implemented in the SYBYL program. Non-cross validated $\left(\mathrm{r}^{2}\right)$ values were determined for the models using linear regression analysis (with variances reported as the standard error of estimation, SEE) which is considered significant when $\mathrm{r}^{2}$ is greater than 0.7. The $\mathrm{q}^{2}$ values obtained were considered significant at 0.3 . The $3 \mathrm{D}$ graphical representation of the steric and electrostatic fields generated through CoMFA is shown with the relative contributions of these fields represented as a 3D coefficient map with favored 80\% steric (green) and electro-static (blue) effects and 20\% disfavored steric (yellow) and electro-static effects (red). Green colored areas of the map indicate where sterically bulky groups may enhance interaction affinity. Blue colored areas $(80 \%)$ indicate regions where a more positively charged group will likely lead to increased binding affinity, while red areas indicate where a more negatively charged group will likely lead to increased binding (20\%). Biological data were taken from the literature ${ }^{12,15^{-18}}$ and entered as $\mathrm{pK}_{\mathrm{i}}$ values in the spreadsheets accessed by the CoMFA routine in SYBYL (Table 1).

The results from the CoMFA study are shown in Figure 2 and summarized in Table 2. This model gave a good cross-validated regression $\left(\mathrm{q}^{2}\right)$ of 0.6 for the training set compounds. Statistically significant models usually are expected to yield $\mathrm{q}^{2}$ xcess of 0.3. The non-cross validated regression of the CoMFA model was found to be 0.9 (Table 3). In this model, electrostatic contributions played a more significant role than steric contributions, with contributions of $61 \%$ and $39 \%$, respectively. 


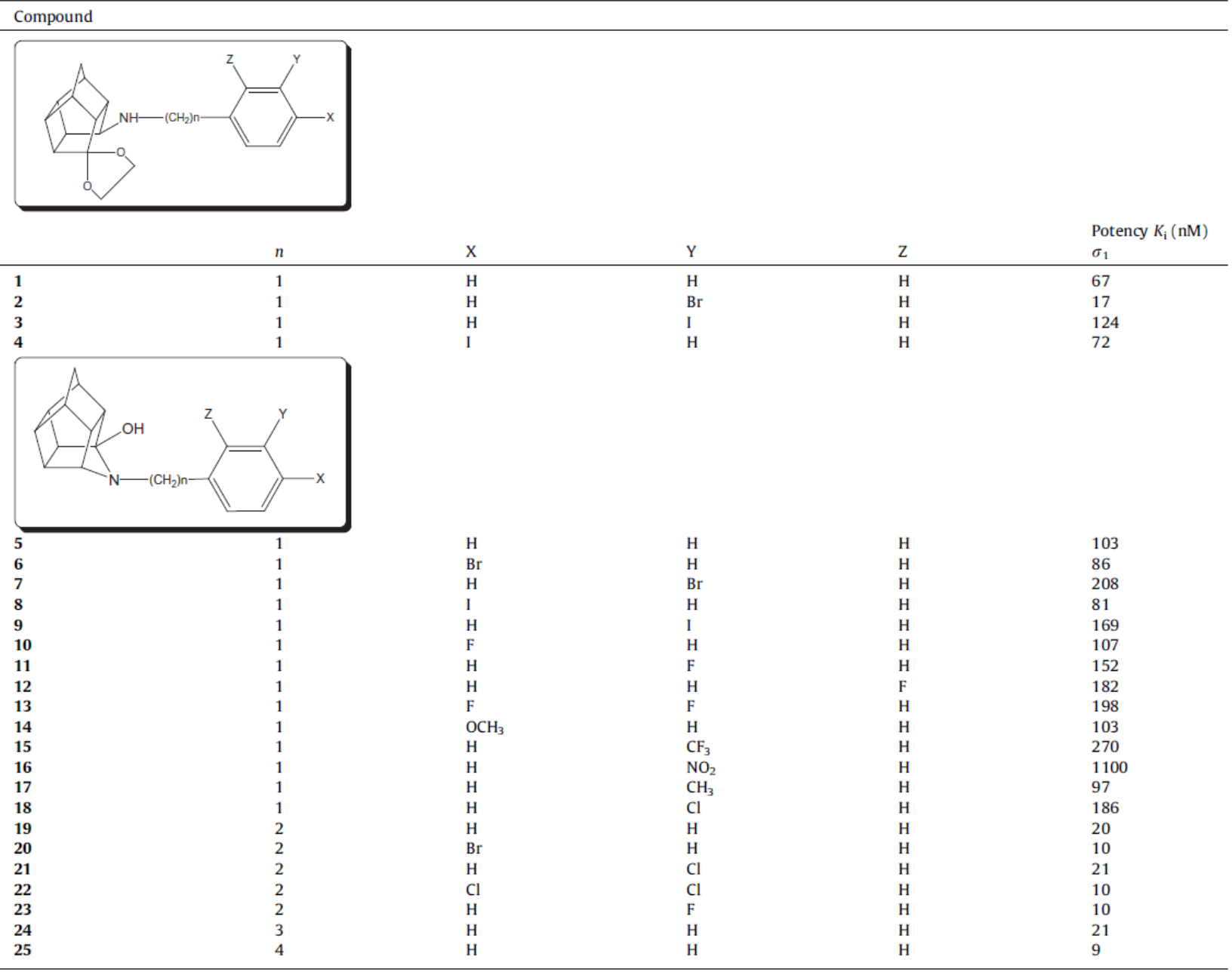

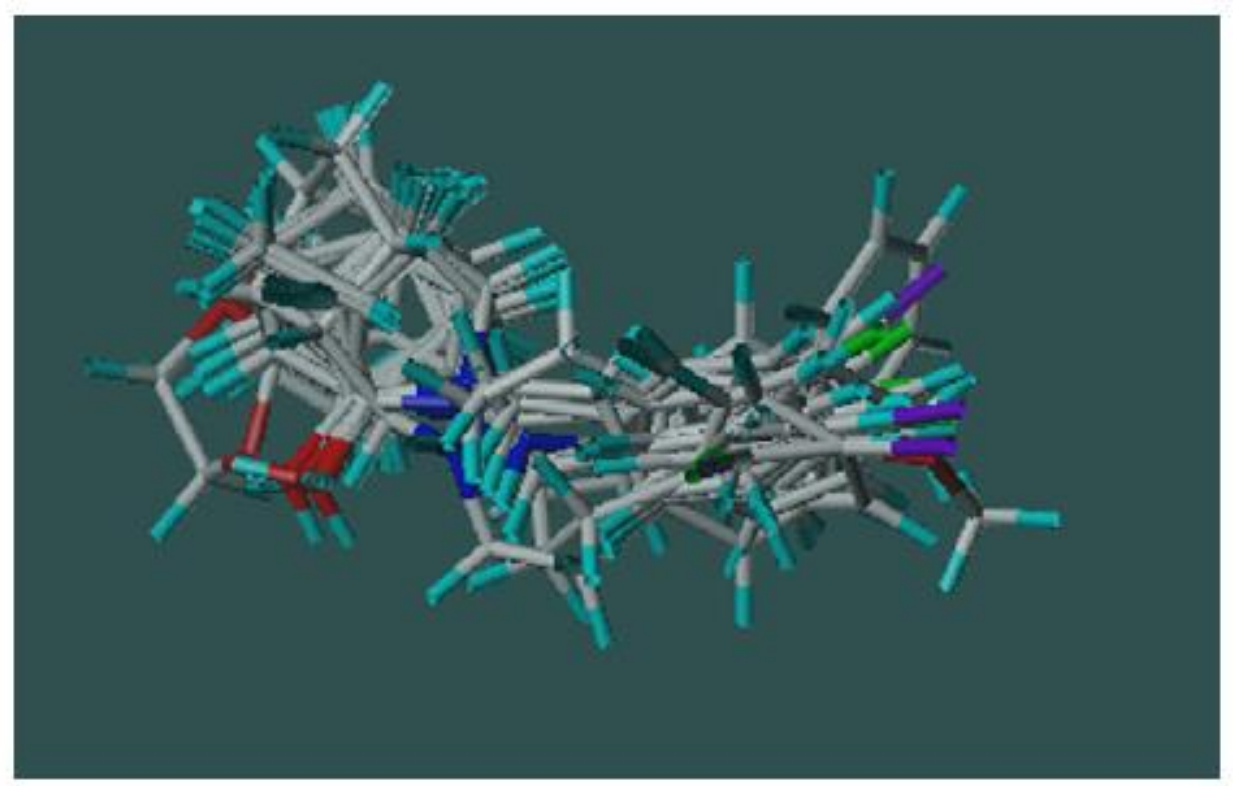

Figure 1. Alignment of the compounds. 
Table 2

Results from the CoMFA and CoMSIA analysis

\begin{tabular}{llllllll}
\hline & & & & & & \multicolumn{2}{c}{ Fraction } \\
\cline { 6 - 8 }$q^{2}$ & SEP & NC & $r^{2}$ & SEE & $F$ & Steric & Electrostatic \\
\hline 0.686 & 0.334 & 5 & 0.986 & 0.071 & 195 & 0.386 & 0.614 \\
\hline
\end{tabular}

$q^{2}$ is the cross-validated regression correlation coefficient; $r^{2}$ is the non-cross validated regression coefficient; SEP is standard error of prediction; NC is number of components used in PLS analysis; SEE is standard error of estimate; $F$ is the Fischer ratio.

This is to be expected, since the aromatic substitution pattern in the training set heavily favored halogens that all influence the electronic character of the aromatic ring. To validate the CoMFA model, the activities of the test set compounds were predicted (Table 4). The CoMFA model was able to predict the activities of the test set compounds with an $r^{2}$ of 0.78 , suggesting the model to be reliable also in predicting other similar cage compounds' activity at the sigma-1 receptor (Fig. 3).

The contour maps of the CoMFA model with compound 5 are shown in Figure 2. As can be seen from this figure, the contour maps correlate with the steric (39\%) and electrostatic (61\%) contributions calculated in the CoMFA model. Green maps indicate areas where increased bulk (i.e., adding larger groups) to the molecule would likely lead to an increase in activity, while the yellow areas indicate areas that are sterically hindered, that is where increasing steric bulk would likely lead to a decrease in activity. Two green maps can be seen, with a larger map located in close proximity to the aromatic ring of the compounds and a smaller map located near the $\mathrm{C}_{1}-\mathrm{C}_{2}$ chain between the cage-amine and the aromatic ring. A large yellow map is located close to the green map. Taking these steric maps into consideration, it is clear that the substitution pattern on the aromatic ring significantly influences the activity of these compounds. 
5

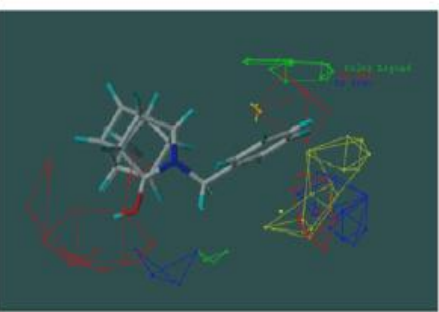

23

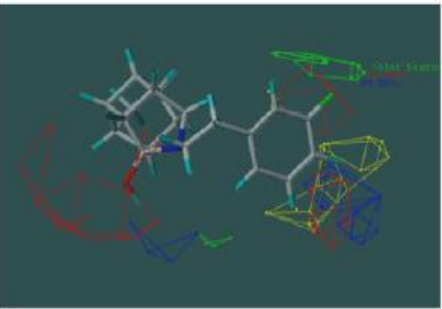

15

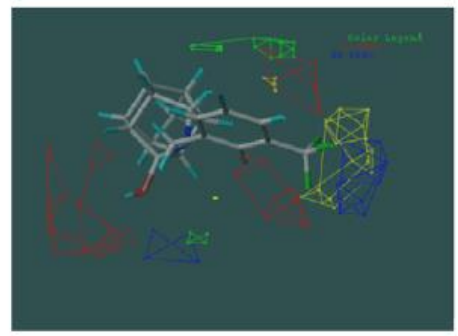

4

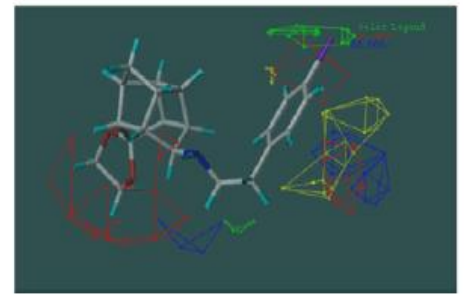

20

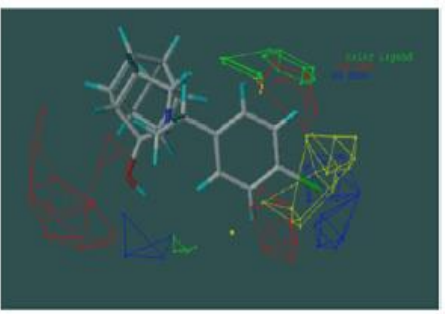

16

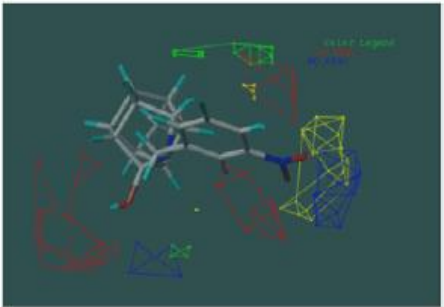

24

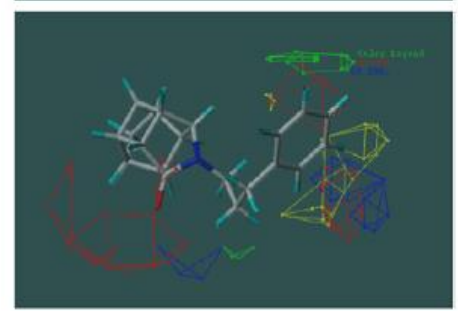

Figure 2. Contour maps of the CoMFA modeling. Several of the compounds (numbers corresponding to the structures in Table 1) are shown within the maps to illustrate the correlation between activity and structure. Green steric maps indicate areas where increasing steric bulk would likely increase activity, while yellow areas delineate where bulky groups likely will decrease activity. Electrostatic maps are shown in red and blue. Blue maps indicate areas where a positive charge will likely increase activity and red areas where a negative charge will increase activity. 
Table 3

Predicted sigma-1 receptor binding for the training set compounds

\begin{tabular}{lllr}
\hline Compound & $\mathrm{p} K_{\mathrm{i}}$ & PREDCOMFA & Residual \\
\hline $\mathbf{1 9}$ & 7.70 & 7.75 & 0.05 \\
$\mathbf{2 0}$ & 8.00 & 8.01 & 0.01 \\
$\mathbf{2 1}$ & 7.68 & 7.75 & 0.07 \\
$\mathbf{2 3}$ & 8.00 & 7.97 & -0.03 \\
$\mathbf{2 4}$ & 7.68 & 7.66 & -0.02 \\
$\mathbf{5}$ & 6.99 & 6.92 & -0.07 \\
$\mathbf{1}$ & 7.17 & 7.08 & -0.10 \\
$\mathbf{3}$ & 6.91 & 6.98 & 0.07 \\
$\mathbf{4}$ & 7.14 & 7.22 & 0.07 \\
$\mathbf{6}$ & 7.07 & 7.02 & -0.05 \\
$\mathbf{7}$ & 6.68 & 6.67 & -0.01 \\
$\mathbf{9}$ & 6.77 & 6.68 & -0.09 \\
$\mathbf{1 0}$ & 6.97 & 6.95 & -0.03 \\
$\mathbf{1 1}$ & 6.82 & 6.77 & -0.04 \\
$\mathbf{1 2}$ & 6.74 & 6.69 & -0.05 \\
$\mathbf{1 3}$ & 6.70 & 6.70 & 0.00 \\
$\mathbf{1 4}$ & 6.99 & 7.01 & 0.02 \\
$\mathbf{1 5}$ & 6.57 & 6.62 & 0.05 \\
$\mathbf{1 6}$ & 5.96 & 6.09 & 0.13 \\
$\mathbf{1 7}$ & 7.01 & 7.03 & 0.02 \\
\hline
\end{tabular}

Table 4

Predicted activities of the test set compounds

\begin{tabular}{lllr}
\hline Compound & $\mathrm{p} K_{\mathrm{i}}$ & PREDCOMFA & Residual \\
\hline $\mathbf{2}$ & 7.77 & 7.02 & -0.75 \\
$\mathbf{8}$ & 7.09 & 7.09 & 0.00 \\
$\mathbf{1 8}$ & 6.73 & 6.48 & -0.25 \\
$\mathbf{2 2}$ & 8.00 & 7.72 & -0.28 \\
$\mathbf{2 5}$ & 8.05 & 7.70 & -0.34 \\
\hline
\end{tabular}

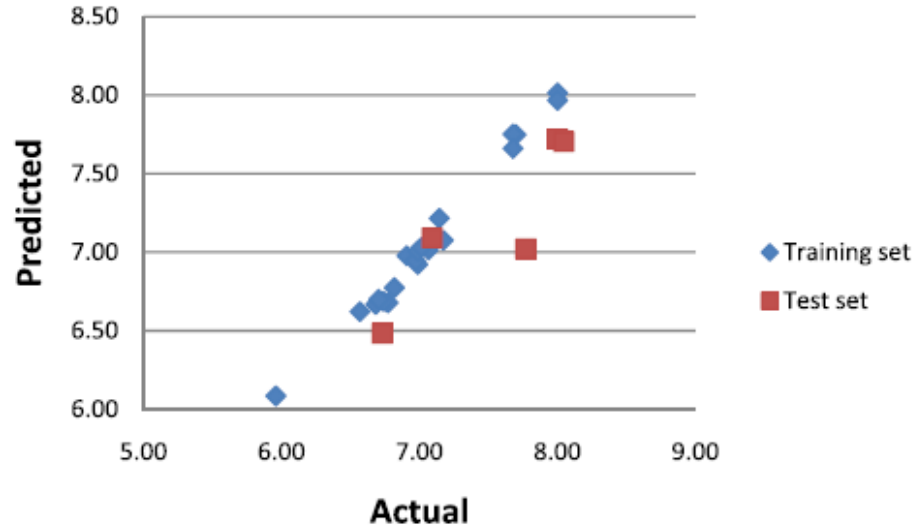

Figure 3. Correlation between the actual $\mathrm{p} K_{\mathrm{i}}$ values and the predicted $\mathrm{p} K_{\mathrm{i}}$ values from the CoMFA model, for both the training and test sets.

Even though CoMFA maps should not be over interpreted as 'receptor' maps, it may suggest that the substituents are generally interacting with a specific grouping of amino acid residues in the sigma-1 receptor, and that larger groups in this region are likely to decrease interaction. Electrostatic maps are shown in red and blue, indicating 
areas where negative and positive charges respectively, would likely increase activity. These aza-cage amines all share an oxygen moiety near the aza-bridge head. For example in compound 5, and seen in Figure 2, a large red map surrounds these oxygen atoms, while two smaller red maps are located close to the aromatic ring (e.g., compound 5), correlating with substituent positions. The blue map correlates with the aza nitrogen of the compounds, and supports the observation that compounds without the aza-bridge have limited or no activity for the sigma-1 receptor. ${ }^{16}$

For compounds 20 and 23, which are the most potent binders to sigma-1 receptor compounds in this series, it can be seen that their respective aromatic rings are oriented towards the green contour, which would indicate an increased activity (Fig. 2). In contrast to this, in the same series compounds 15 and 16 exhibit the lowest affinity for binding to the sigma-1 receptor, which could be explained by the projection of the $\mathrm{NO}_{2}$ - and $\mathrm{CF}_{3}$-groups into the large yellow sterically disfavored area. Additionally, for compound 16 , the oxygen atoms of the $\mathrm{NO}_{2}$ - group extend towards a blue map that could also explain its lack of activity at the sigma-1 receptor. The activity of compounds 4 and 24 can also be explained by the CoMFA model by the fact that the carbon-chain connecting the cage with the aromatic ring extends towards a green map.

To gain insight into the interaction between this series of pentacycloundecylaminederived compounds and the sigma-1 receptor, we developed a homology model. Homology modeling was done using YASARA structure version 10.10.4. ${ }^{19,20}$ We chose to use YASARA based on its impressive performance at CASP8, an international competition for the prediction of protein structure (see Ref. 21 for further information on the methodology of how YASARA performed at CASP8, as well as the methods of homology modeling, 20 and also at YASARA's website: www.yasara.org). The resultant homology model was based on the template structure recently reported. ${ }^{22}$ The receptor homology was based on protein code 3CIA. For the docking study, we used MOE-Dock (www.chemcomp.com). The protein was protonated at $\mathrm{pH} 7.4$, to assure biological relevance. As can be seen in Figure 4, the pentacycloundecylamines docked into two distinct, but closely proximal sites (Fig. 4A). A pocket deeper into the protein, as well as one closer to the surface is evident. Docking studies suggest that for compound $19(\mathrm{Ki}=20 \mathrm{nM})$, the aromatic ring is able to orient towards the inner part of the receptor (Fig. 4B) for possible hydrophobic interactions. This orientation is in contrast with data found for compound $16\left(\mathrm{~K}_{\mathbf{i}}=\right.$ $1100 \mathrm{nM}$ ), where the $-\mathrm{NO}_{2}$ moiety is oriented towards the solvent interface (Fig. $4 \mathrm{C}$ ), leaving only the cage unit and possibly the secondary amine and hydroxyl groups to undergo hydrogen bonding. As seen from Figure $4 \mathrm{D}$, compound 18 shows a probable hydrogen bonding opportunity between the secondary amine, hydroxyl and MET1, indicating that this binding mode may play a role in activity. To validate that the aza nitrogen is important in binding as shown in the docking study, we tested two 
oxa-PCU-derived compounds, NGP1-01 and its phenethyl derivative 23,24 for $[3 \mathrm{H}]-$ pentazocine binding in rat liver homogenate (Fig. 5). As can be seen from the binding data, there is a >10-fold decrease in activity for the binding of the oxa compounds as compared to the aza compounds. Taken together with the docking data, this suggests that the aza orientation is be generally preferred for binding to the sigma1 receptor. The importance of the nitrogen in binding to the sigma-1 receptor corroborates in part previous pharmacophore work published, which suggested that at a minimum, one nitrogen was important for binding of phenylalkylpiperidines and phenylalkylpiperazines. A previous pharmacophore for the sigma-receptor was suggested to be $\mathrm{C}-\mathrm{N}(\mathrm{R})-\mathrm{X}-\mathrm{Ph}$. Additionally, the $\mathrm{C}$ and aromatic moiety ( $\mathrm{Ph}$ ) from the pharmacophore are located in areas of bulky hydrophobic tolerance. 25 This presence of two hydrophobic pockets may accommodate several binding modes for a single compound. ${ }^{25,26}$ From the current study, it can be extrapolated that the polycyclic cage may prove useful in future studies to delineate binding to these two hydrophobic pockets, and therefore binding modes, as compared to the use of only aromatic rings as seen in many of the compounds published.

In conclusion, in this study, we have shown a $3 \mathrm{D}-\mathrm{QSAR}$ model for azapentacycloundecylamine-derived compounds at the sigma-1 receptor. The conclusion from this model is that the substitution pattern on the aromatic ring has a low tolerance for sterically large groups, with the electrostatic nature of the substituent further determining binding in these structures.

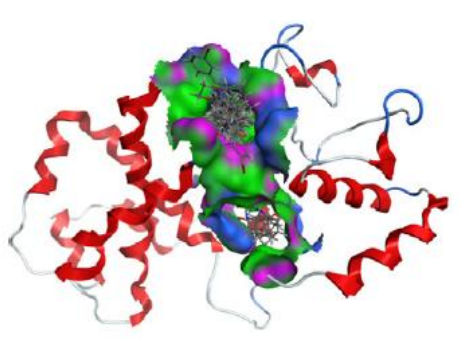

A

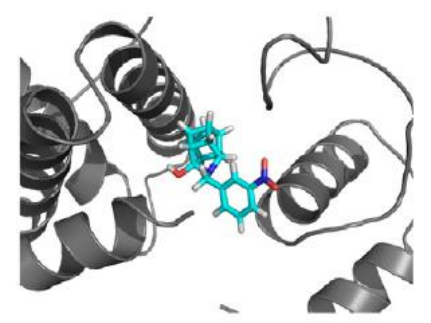

C

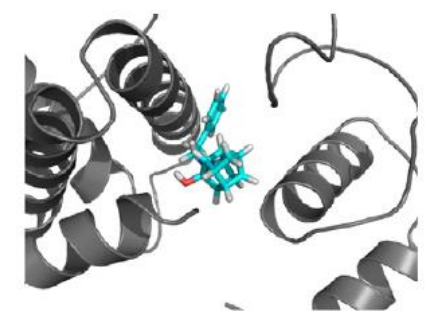

B

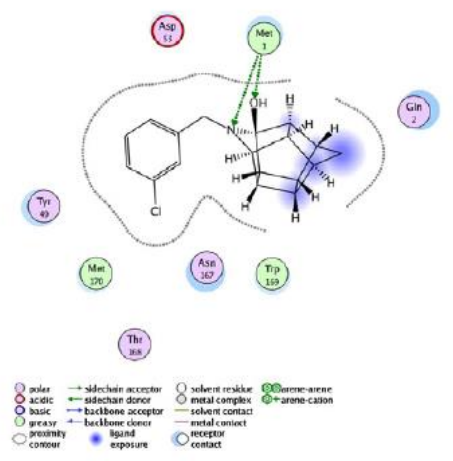

D

Figure 4. (A) Docking results from MOE suggested two main docking sites; (B) Compound 19 docked into the receptor; (C) Compound 16 docked into the receptor; (D) Interaction diagram between compound 18 and the receptor showing possible important hydrogen bonds. 


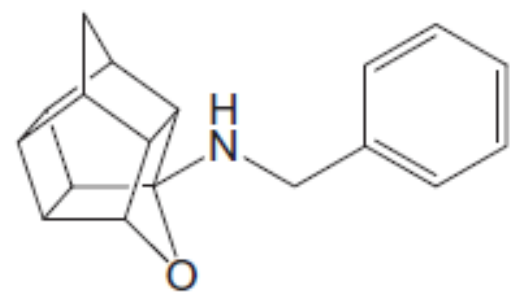

NGP1-01:

$\mathrm{IC}_{50}=1.78 \mu \mathrm{M}$

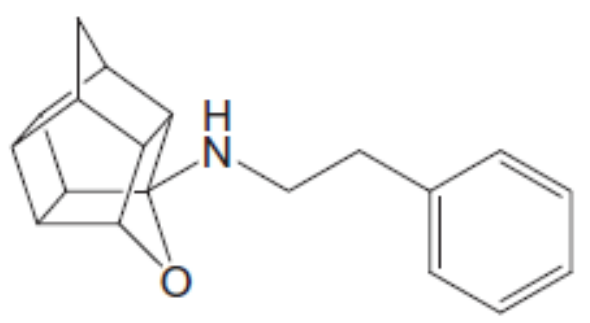

Phenethyl derivative of NGP1-01:

$\mathrm{IC}_{50}=1.54 \mu \mathrm{M}$

Figure 5. Structures and sigma-1 receptor binding of oxa-PCU-derived compounds.

Here too we show docking data, where two main clusters of docked compounds in a putative binding site are shown. With the pentacycloundecylamine-derived compounds showing promise as lead structures in a variety of psychiatric and neurodegenerative diseases, the current model could be used to develop novel compounds within this class.

\section{Acknowledgement}

This work was funded in part by the Bloomberg Ophthalmology Fund, Youngstown $\mathrm{OH}$ to W.J.G. 


\section{References and notes}

1. Monnet, F. P. Biol. Cell 2005, 97, 873.

2. Quirion, R.; Bowen, W. D.; Itzhak, Y.; Junien, J. L.; Musacchio, J. M.; Rothman, R. B.; Su, T. P.; Tam, S. W.; Taylor, D. P. Trends Pharmacol. Sci. 1992, 13, 85 .

3. Monnet, F. P.; de Costa, B. R.; Bowen, W. D. Br. J. Pharmacol. 1996, 119, 65. 4. Hayashi, T.; Su, T. P. Cell 2007, 131, 596.

4. Hanner, M.; Moebius, F. F.; Flandorfer, A.; Knaus, H. G.; Striessnig, J.; Kempner,

5. E.; Glossmann, H. Proc. Natl. Acad. Sci. U.S.A. 1996, 93, 8072.

6. Hellewell, S. B.; Bowen, W. D. Brain Res. 1990, 527, 244.

7. Su, T. P.; Hayashi, T.; Maurice, T.; Buch, S.; Ruoho, A. E. Trends Pharmacol. Sci. 2010, 31, 557.

8. Hayashi, T.; Su, T. P. J. Pharmacol. Exp. Ther. 2003, 306, 726.

9. van Waarde, A.; Ramakrishnan, N. K.; Rybczynska, A. A.; Elsinga, P. H.; Ishiwata, K.; Nijholt, I. M.; Luiten, P. G.; Dierckx, R. A. Behav. Brain Res. 2011, 221, 543.

10. Mavlyutov, T. A.; Nickells, R. W.; Guo, L. W. Mol. Vis. 2011, 17, 1034.

11. Smith, S. B.; Duplantier, J.; Dun, Y.; Mysona, B.; Roon, P.; Martin, P. M.; Ganapathy, V. Invest. Ophthalmol. Vis. Sci. 2008, 49, 4154.

12. Nguyen, V. H.; Kassiou, M.; Johnston, G. A.; Christie, M. J. Eur. J. Pharmacol. 1996, 311, 233.

13. Liu, X.; Nuwayhid, S.; Christie, M. J.; Kassiou, M.; Werling, L. L. Eur. J. Pharmacol. 2001, 422, 39.

14. Geldenhuys, W. J.; Bezuidenhout, L. M.; Dluzen, D. E. Eur. J. Pharmacol. 2009, 619, 38.

15. Liu, X.; Banister, S. D.; Christie, M. J.; Banati, R.; Meikle, S.; Coster, M. J.; Kassiou, M. Eur. J. Pharmacol. 2007, 555, 37.

16. Liu, X.; Kassiou, M.; Christie, M. J.; Hambley, T. W. Aust. J. Chem. 2001, 54, 31.

17. Kassiou, M.; Nguyen, V. H.; Knott, R.; Christie, M. J.; Hambley, T. W. Bioorg. Med. Chem. Lett. 1996, 6, 595.

18. Liu, X.; Kassiou, M.; Christie, M. J. Aust. J. Chem. 1999, 52, 209.

19. Venselaar, H.; Joosten, R. P.; Vroling, B.; Baakman, C. A.; Hekkelman, M. L.; Krieger, E.; Vriend, G. Eur. Biophys. J. 2010, 39, 551.

20.Krieger, E.; Koraimann, G.; Vriend, G. Proteins 2002, 47, 393.

21. Krieger, E.; Joo, K.; Lee, J.; Raman, S.; Thompson, J.; Tyka, M.; Baker, D.; Karplus, K. Proteins 2009, 77, 114.

22. Laurini, E.; Dal Col, V.; Mamolo, M. G.; Zampieri, D.; Posocco, P.; Fermeglia, M.; Vio, L.; Pricl, S. ACS Med. Chem. Lett. 2012, 2, 834.

23. Geldenhuys, W. J.; Terre'Blanche, G.; Van der Schyf, C. J.; Malan, S. F. Eur. J. Pharmacol. 2003, 458, 73.

24. Geldenhuys, W. J.; Malan, S. F.; Murugesan, T.; Van der Schyf, C. J.; Bloomquist, J. R. Bioorg. Med. Chem. 2004, 12, 1799. 
25. Glennon, R. A. Mini-Rev. Med. Chem. 2005, 5, 927.

26. Ablordeppey, S. Y.; Fischer, J. B.; Glennon, R. A. Bioorg. Med. Chem. $2000,8,2105$.

27. Hawkins, P. C.; Skillman, A. G.; Warren, G. L.; Ellingson, B. A.; Stahl, M. T. J. Chem. Inf. Model. 201050

28. Hawkins, P. C. D.; Nicholls, A. J. Chem. Inf. Model. 2012, 52, 2919. 